\title{
Noch einiges zum ägyptischen Neter.
}

Von Professor D. Dr. Karl Beth in Wien.

Es freut mich, daß meine Abhandlung über El und Neter (36. Jahrgang, S. 129-I86) eine Erwiderung vom Gesichtspunkte der Ägyptologie hervorgerufen hat. Allerdings hätte ich gewünscht, daB ein umsichtigerer und dem behandelten Stoffe näher stehender Vertreter dieser Wissenschaft zu meinen Ausführungen Stellung genommen hätte. Denn da $\beta$ Herrn DR. GRAPOWs Feder hierzu nicht berufen war, geht zur Genüge aus der ganzen Anlage und Haltung der (im folgenden Jahrgang, S. I99-208) ihr entflossenen Erwiderung hervor, die nur dadurch erklärt werden kann, $\mathrm{da} B$ G. des Verständnisses für religionsgeschichtliche Fragestellungen ebenso ermangelt wie der Fähigkeit, auf solche einzugehen. Nur von hieraus wird es verständlich, daß er, statt meine These zum Gegenstande der Erörterung $\mathrm{zu}$ machen, es vorgezogen hat, $\mathrm{zu}$ explizieren, daß ich kein Ägyptolog vom Fach bin - was die Leser dieser Zeitschrift ohnedies wissen. Er hat aber gar nicht für nötig erachtet, die sachlichen Gründe, die ich beibringe, zu besprechen, geschweige denn das Problem, um das es sich handelt, auch nur im mindesten $z u$ beleuchten; und das ist in diesem Falle eine um so mehr irreführende Unterlassung, als G. dadurch bei seinen Lesern den Eindruck erweckt, als stehe der Gegenstand meiner Erörterung außerhalb desjenigen Rahmens von Fragestellungen und Argumentationen, den angesehene Ägyptologen von Fach selbst benützen.

Ich muß allerdings bemerken, daß ein Problem, wie das von mir angeschnittene, dem in philosophischen und ethnologischen Dingen Unbewanderten immer etwas Fremdartiges bleiben wird. Handelt es sich hier doch um die scharfe Umgrenzung der Nüancen eines Begriffs, den wir bisher alle als etwas Gegebenes und Eindeutiges hingenommen haben. Das ist er jedoch nicht. Daß er es nicht ist, kann freilich nur der einsehen, der mit einem durch religionsgeschichtliche und religionspsychologische Vergleichung geschulten Blick das hieher gehörige Material be- 
trachtct. Bei abstrakten und ideologischen Begriffen ist natürlich die genaue Beobachtung des Bedeutungswandels viel schwieriger als bei Dingbegriffen, deren im Entwicklungsprozeß der Volks- und Völkerpsyche sich abspielender Bedeutungswechsel häufig klar zutage liegt. Gleich hier möchte ich deshalb behufs trefflicher Illustration, um nicht immer von Eigenem zu reden, auf die mustergültige Untersuchung N. SODERBLOMs über die Bedeutung des Wortes "heilig" aufmerksam machen sowie auf seinen Hinweis, wie stark noch in der Verwendung, die dieser Begriff bei KANT erfährt, die primitiven Motive nachwirken'. Allenthalben liegen unbewußter Weise in der Sprache wie im Empfinden prähistorische und auch „prälogische" Vorstellungen vor, denen es nachzugehen gilt. Das Ergebnis solcher Untersuchungen kann wichtig werden, indem es entweder, wie bei SODERBLOM, dazu dient, das Problematische und Bedingte unserer heutigen Vorstellungen und Begriffe uns zu Gemüte zu führen, oder indem es, wie es mir vorschwebt, einen Einblick nicht. in die Mythologie und Dogmatik eines Volkes, sondern in sein Empfindungsund Vorstellungsleben eröffnet. Hierfür kommt es eben darauf an, festzustellen, welche Klangfarbe ein schon seit je bekannter Ton unter verschiedenen Bedingungen besitzt, oder welcher Grundton sich aus einem verworren an unser $\mathrm{Ohr}$ dringenden Akkord ermitteln läßt. Es wirkt daher unwillkürlich lächerlich, wenn sich $G$. angesichts meiner in dieser Richtung unternommenen Bemühungen immer wieder letztlich auf die Behauptung zurückzieht, nețer heißt Gott. 'Ich hatte freilich, das gestehe ich ganz offen, den entschiedensten Protest gegen meinen Lösungsversuch des Neterproblems erwartet. Ja ich mußte ihn sogar auch in der Form völliger Ablehnung der Problemstellung selbst erwarten, seit mir im Sommer igr5, während ich mit der Abhandlung beschäftigt war, ein deutscher Ägyptologe, dem ich von meiner Arbeit erzählte, keinen weiteren Beitrag für meine Untersuchung $z u$ geben wußte als den: „Neter heißt Gott!" Und doch würde selbst dann, wenn wir neter immer mit Gott übersetzen müßten, kein Völkerpsycholog oder Religionshistoriker annehmen, daß die alten Ägypter bei diesem Wort dasselbe gedacht hätten wie wir oder wie die alten Germanen. Auf die spezifische Begriffsnüance kommt es aber an. Wie diese bei den alten Ägyptern beschaffen war, beziehungsweise auf welche Grundvorstellung sie zurückgeht, das wissen wir bis heute nicht, und deshalb

I N. Söderblom, Das Werden des Gottesglaubens, passim, bes. S. I95 ff. $212 \mathrm{f}$. 
schrieb ich meine Abhandlung als einen Beitrag zu dieser Frage. Der darin vorgelegte Versuch einer Lösung des Problems mag ja vielleicht, wie seine Vorgänger, aus sachlichen Gründen für ungenügend erklärt werden; ihn aber grundsätzlich, und nur grundsätzlich abzulehnen, das muß jedem, der einmal das Schwergewicht dieses Problems gespürt 'hat, ein Lächeln abnötigen. Und doppelt lächerlich wirkt G.s Entrüstung über meine Problemstellung, wenn man bedenkt, daß dieses Problem schon viele Ägyptologen, deutsche wie englische und französische, beschäftigt hat.

Allein der eben erwähnte knappe Ausspruch über die sichere Bedeutung von $n \underline{t} r$ ist deshalb besonders erwähnenswert, weil in ihm jene Apodiktizität recht majestätisch zum Ausdruck kommt, die auch G. in seiner Erwiderung taktisch anzuwenden bestrebt ist, während er sie mir unbilliger Weise zum Vorwurfe macht. Denn eben G. ist es, der mit kurzen runden Behauptungen aufwartet, wo er als Mann der Wissenschaft mit einem "vielleicht", "wahrscheinlich" oder „etwa" operieren sollte. $\mathrm{Da} \beta$ das Neter-Problem tatsächlich existiert, daß es in der ägyptologischen Wissenschaft anerkannt ist und wie schwierig die Frage nach der Grundbedeutung von neter ist, dies mag man daraus ersehen, daß zahlreiche namhafte und hoch verdiente Forscher ihre Aufmerksamkeit diesem Gegenstand zugewendet und zu seiner Bewältigung die eine oder andere Hypothese gebildet haben. Ich hatte die Leser meiner Abhandlung mit diesem wissenschaftsgeschichtlichen Stoffe nicht behelligen wollen, sehe mich aber jetzt genötigt, zur Beleuchtung der Streitfrage aus der Fülle der Arbeiten über nețer einiges herauszugreifen, wodurch ohne weiteres einleuchtet, wie es um das dictum "Neter heiBt Gott" bestellt ist. PIERRET befürwortete 1879 im Essai sur la Mythologie Égyptienne die Grundbedeutung „Erneuerung" und erkannte in dem ägyptischen Gottesbegriff den Grundgedanken der ewigen Selbstverjüngung. LEPAGE-RENOUF beklagte in seinen Hibbert Lectures von IS79 on the Origin and Growth of Religion as illustrated by the Religion of Ancient Egypt, daB bisher noch keine befriedigende Erklärung von nețer gegeben sei, und fand seinerseits den Grundbegriff "mighty", "might", "strong", "power", ,which is also the meaning of the Hebrew El". BRUGSCH kombinierte gewissermaßen beide Ansichten; -indem er sich jedoch mehr an PIERRET anschloB und als Grundbedeutung von nẹter erklärte "die tätige Kraft, welche in periodischer Wiederkehr die Dinge erzeugt und erschaff, ihnen neues Leben verleiht und die Jugend- 
frische zurückgibt". Ähnlich stellte sich DE ROUGÉ im dritten Band seiner Chrestomathie. Nach alledem machte MASPERO einen Rückzug, betonte aufs neue, daß bislang der Ursinn von neter nicht feststellbar sei, ohne jedoch den Hinweis zu unterlassen, daß die in historischer Zeit gewöhnliche Bedeutung Gott nicht die ursprüngliche ist ${ }^{\mathrm{I}}$. Sodann ist AMÉLINEAU im Jahre $1908 \mathrm{mit}$ einem sehr bedeutsamen größeren Werke von 536 Seiten auf den Plan getreten, das sich gänzlich dem $n \underline{t} r$-Problem widmet. Aus folgendem Satze der Vorrede ist ersichtlich, wie aktuell dem Verfasser dieses Problem erscheint: C'est cette étude du sens attaché par les Égyptiens au mot Dieu qui fait l'unité de mon livre : il n'est pas un seul chapitre de cet ouvrage dont toutes les idées, ne convergent vers le sens à attribuer à ce mot quoique l'étude de ce sens ne vienne qu'au quatrième chapitre ${ }^{2}$. Er erklärt die Bedeutung Dieu ausdrücklich für den sens dérivé von $\rceil^{3}$, lehnt die Bedeutungen fort und rajeuni ab und sucht festzustellen, daß nțr ursprünglich „eine besondere Klasse von Wesen" bezeichnet, nämlich die Toten; und er kommt andererseits mittels der Annahme eines uralten ägyptischen Totemismus $z u$ dem Schluß, die eigentliche Bedeutung von $n \underline{t} r$ sei „Beschützer". Unstreitig hat AMÉLINEAU in diesem Werke einen höchst beachtlichen Beitrag zur Lösung des $n t \underline{t} r$-Problems geliefert; nur ist es mit seinen Feststellungen noch nicht erledigt. Denn wenn auch die Toten als ntruy bezeichnet werden, so ist das eben wohlgemerkt eine Bezeichnung, 'die 'selbst erst einer, Erklärung harrt, sofern“ zu untersuchen ist, weshalb das Wort $n \underline{t} r$, das auch die Götter bezeichnet und zudem in manch anderer Verbindung gebraucht wird, zur generellen Charakterisierung der Toten wie auch zur Benennung eines einzelnen Verstorbenen angewandt wird. Diese Frage könnte nur beantwortet werden, wenn die Urbedeutung von $n \underline{t} r$ zu ermitteln wäre. Dieselbe mag nun derjenigen von "Beschützer" in der Tat nahe kommen; jedoch ist diese zu speziell, auch in gar zu vielen Fällen nicht brauchbar, als daß sie die Grundbedeutung des Wortes sein könnte.

Ich begnüge mich mit diesem Überblick, der die Wirklichkeit und Wichtigkeit des ntr-Problems hinlänglich dartut, zugleich aber auch zeigt, daß alle namhaften Ägyptologen, die sich um die ägyptische

I Vgl. G. MASPERo, La mythologie Égyptienne in Biblioth. Égyptol. II, p. 215 f.

${ }^{2}$ E. Amélinenu, Prolégomènes à l'Étude de la religion Égyptienne, p. II.

${ }^{3}$ P. 182. 
Religionsgeschichte verdient gemacht haben, nicht in der Lage gewesen sind, sich bei der Bedeutung "Gott" für $n \underline{t}, *$ u beruhigen. In der Tat liegt hier ein Problem von ganz fundamentaler Bedeutung vor, welches noch immer neu in Angriff genommen werden muß, auch gegen den Protest schnell befriedigter Gemüter. Daß hierbei, da philologische Mittel versagen, andere Wege gegangen werden müssen, ist nur natürlich, und hier eben tritt die vergleichende allgemeine Religionsgeschichte in eine Lücke ein. Der Unwille des zünftigen Herrn G. darüber, daß ein Nichtägyptologe, sogar ein Theologe sich mit dieser Frage beschäftigt, nimmt sich doch gar zu kläglich aus angesichts des sonst in der wissenschaftlichen Welt allgemein gepflegten Zusammenund Ineinanderarbeitens sowie des Tatbestandes, daß die religionsgeschichtliche. Forschung geradezu berufen, ist, bei Fragen der religiösen Interpretation dort ihre bescheidenen Beiträge zur Verfügung zu stellen, wo die Philologie versagt. Auch nicht als Theologe, sondern eben als Religionshistoriker habe ich das Wort ergriffen. Als solcher führe ich es weiter. Die von den Gesichtspunkten der anderen Wissenschaft aus unternommene Nachprüfung mag alsdann entscheiden, ob der von religionsgeschichtlichen Erwägungen aus gesteuerte Beitrag eine Förderung des Problems bedeutet oder nicht. Aber dagegen muß entschiedenste Verwahrung eingelegt werden, daß jemand unter Ablehnung einer sachlichen Nachprüfung an einigen nebensächlichen Punkten herumkritelt und damit den Gedankengang und die These einer Abhandlung für abgetan erklärt. Da G. die Nachprüfung unterlassen hat, so konstatiere ich, daß trotz der Überreiztheit meines Gegners und trotz des Anscheins der Fehlerhaftigkeit, den er meinen Darlegungen aufzudrücken versucht hat, meine These durch ihn gänzlich unberührt stehen bleibt.

Darauf allein soll es mir für jetzt ankommen, da ich unmöglich den Lesern zumuten kann, mit mir in eine langwierige Zurückweisung der einzelnen ungerechten Angriffe G.s einzutreten, bei der nichts Positives gefördert werden kann. Nur streifen möchte ich deshalb vorab die sich an der Peripherie haltenden Bemerkungen G.s. Mit vollem Recht hebt ja G. nachdrucksvoll hervor, daB gleich meine erste Publikation aus dem Gebiete der ägyptischen Religionsgeschichte einen sehr schweren Stoff behandelte. Allein die Fragen der Religionsgeschichte pflegen ihren Bearbeiter nicht an der Oberfläche zu halten; und ich habe das Ägyptische nicht gelcrnit, um die Philologie zu fördern - denn ich bin kein Philo$\log$ - sondern einzig und allein zum $Z_{w e c k}$ religionsgeschichtlicher 
Studien. Trotzdem oder gerade darum hatte ich es nicht für nötig gehalten, in einer fachwissenschaftlichen Zeitschrift so, wie G. es als uncrläBlich vorauszusetzen scheint, über alles, was ich zu erwähnen habe, ab ovo zu handeln; und ich halte G.s Darbietungen über die Aussprache von $n \underline{t r}$ als völlig deplaziert, um nicht zu sagen für lächerlich. Man schreibt ja mit gutem Grund neter, nețer, nuter, nuter, ntr, ohne daß diese oder jene Schreibung erst einer ausdrücklichen Rechtfertigung bedürfte, da eben eine jede ihren guten Sinn hat; wie Wörter gesprochen wurden, wissen wir auch für andere orientalische Sprachen nicht, ohne daß wir deshalb auf Vokalisierung gänzlich verzichten müßten. $D a ß$ bei meinen kurzen Bemerkungen über das $n \underline{t} r$-Determinativ Mißverständliches untergelaufen ist, bedaure ich. Es handelt sich aber bei dem, was ich S. I6o f. darüber sagte, durchaus nicht um "Entdeckungen“, sondern um ganz Bekanntes. Ich habe keineswegs geschrieben, daß schon in den Pyramidentexten zur Determinierung von Göttern vorzugsweise das $n t \underline{t} r$-Ideogramm $ๆ$ diene. Ich habe vielmehr gesagt, daf in den Pyramiden viele Gottheiten gar nicht determiniert werden, daß das beliebteste Determinativ für eine Gottheit überhaupt das Ideogramm? ist und da $\beta^{\prime}$ wir daneben finden, daß in den Mastaba- und Pyramidentexten am häufigsten der Falke auf der Tragstange als Gottesdeterminativ verwendet wird, für weibliche Gottheiten die Uräusschlange oder der Korb mit derselben. Im übrigen neige ich unter den mancherlei versuchten Deutungen des F-Zeichens - die ich (gegen G.! S." I6r) einfach nebeneinander stellte mit den Worten "ob es nun als Axt oder als Fahne oder als Machtsymbol zu deuten sein mag" - derjenigen $\mathrm{zu}$, welche darin eine Axt erblickt - worin mich vor allem die zahlreichen religionsgeschichtlichen Analogien bestärken.

In völligem Irrtum befindet sich G. mit seinen Sätzen über p’̀nt und pśdt. Man fragt sich verwundert, wie ein Forscher, dem die Bearbeitung des ungeheuren Materials des ach wie lange schon erwarteten "Berliner Wörterbuchs" anvertraut sein soll, die Beschäftigung mit der ägyptologischen Fachliteratur also zurückstellen kann. Nicht ich bin es, der "nicht weiß" (S. 202), daß die beiden "Begriffe" pint und pśd $t$ "ja gar nichts miteinander zu tun haben", sondern G. "weiß nicht", daß beide "Begriffe" nicht selten ebenso durcheinander und ineinander gehen wie ihre Schreibungen. Er "weiß nicht", mit welch einem schweren

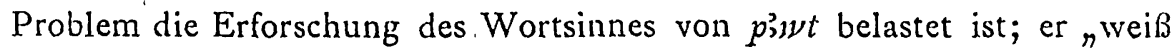


nicht" - denn wüßte er es, so hätte er nicht gegen meine hierauf bezüglichen Sätze polemisieren können -; daß andere Ägyptologen sich mit diesem Problem abgemüht haben und $\mathrm{da} \beta$ eine selbst für den blo $B$ grammatisch und lexikalisch interessierten Philologen sehr bedeutsame Frage die ist, auf welchem Wege gelegentlich diese beiden Begriffe ineinandergeflossen sind. Selbstverständlich „bedeutet" p’nt nicht "Neunheit", wie ich es einmal mit BRUGSCH und mit MORET (letzterer in eben dem fraglichen Satze) wiedergegeben habe. Aber eine seltsame Unberührtheit mit einem wirklichen Problem offenbart sich, wenn G., was in dieser Form geradezu falsch ist, apodiktisch dekretiert: "p $p^{3} \nu t$ bedeutet ,Urzeit', pint als Kollektivum ,Urgötter' und pintj ,zur Urzeit gehörig, Urgott"“. Daß der Begriff "ur" drin steckt, darf als sicher gelten - aber wohlgemerkt: es ist nicht unser ${ }_{n}$ ur", sondern ein davon erheblich abweichender Begriff! Alles .weitere aber ist unsicher, wenigstens nach dem jetzigen lexikalischen Bestande, den wir besitzen. Sicher ist wieder nur, daß $p_{3}^{3} 2 \nu t$ häufig mit Urzeit, $p^{3} 2 \nu t j$ mit urzeitlich und Urgott übersetzt werden kann, ohne daß man jedoch sagen dürfte, dies sei in der Tat der Sinn des Wortes. Die Übersetzung Urgott ist vielmehr eine religionsgeschichtliche Ungeheuerlichkeit angesichts der Verwendung des Wortes pintj (soweit mir dieselbe bisher zugänglich war, füge ich für G. bei; denn die Schatzkammer des „Berliner Wörterbuches" ist leider - quousque tandem - den meisten ein Geheimnis). Ich habe für die Bezeichnung von $p^{3}$ int und $p^{s} \underline{d} t$ manches Material zusammengetragen. Danach dürfte es weiterhin so stehen, daß $p_{22 \nu t j}$ und ntrr zwei in unseren Texten selbständig nebeneinander stehende Bezeichnungen für etwas Göttliches sind und daß die erstere wahrscheinlich zeitlich weiter zurückreicht als die zweite; und hierin stimme ich völlig mit BUDGE ${ }^{I}$ überein. Nun ist aber weiterhin sicher, daß p’nt $(j)$ häufig einen Sinn hat ähnlich dem von pśdt, und ob pśdt selbst von Hause aus Neunheit bedeutet habe, bezw. ob das betreffende Zeichen ${ }_{c}^{\ominus} p \dot{s} \underline{d} t=$ Neunheit zu lesen ist, das ist schon angesichts des Tatbestandes, daß nur eine geringe Zahl der so bezeichneten Göttergemeinschaften gerade aus 9 Göttern besteht, mit Recht von Forschern wie BUDGE in Zweifel gezogen worden. Ich mul das gegen G.s kurze Behauptung, daß ${ }_{0}^{\ominus}$ "Neunheit" heiße, erwähnen, und ich füge hinzu, daß ERMAN in seinem Glossar mit gutem Grund die Lesung pśdt für ${ }_{0}^{\ominus}$ mit einem Fragezeichen

'E. A. Wallis Budge, Osiris and the Egyptian Resurrection (1911), p. 353 . 
versehen hat. Häufig sind, wenn wirklich g Götter gemeint sind, 99 nacheinander geschricben, wic in dem interessanten Wort, das von dem verklärten Könige Unis sagt: „Das Brot seines Mundes ist das Wort des Gottes Geb, das hervorgeht aus dem Munde der neun Götter" (Pyr. Unis 234), ein Spruch, der äußerlich entfernt an Deut $8_{3}$ anklingt. Bei ntrı p’utju kommen wir mit der Fassung „Urzeitgötter" aus; wenn aber pint ntruy [2 09991$]$ geschricben ist, dann muß ein Gedanke vorliegen, der zumindest dem der $p s \underline{d} t$ ähnlich ist; da scheint cine bestimmte Gemeinschaft von Göttern vorgestellt $\mathrm{zu}$ sein oder auch die gesamte Götterschaft, das Pantheon, das Pleroma! Ja man darf crnsthaft erwägen, ob dieser Sinn nicht auch in einer Stelle und Version von Totb. Kap. 77 (nach Pap. des Ani, Z. Ir6) anzunehmen ist: „Hprj (der Schöpfergott), der in seinem Bote wohnt, dessen Leib die pintj ist" Her "Éprj, Urzeitgott, sein eigener Leib". Aber die Übersetzung "dessen Leib p3nt $j$ ist", d. i. die Gesamtheit des Göttlichen, ergibt einen sehr guten Sinn. Denn der Gedanke kehrt oft wieder und wird in den verschiedensten Wendungen ausgedrückt, daß aus éinem Gott alle einzelnen Gottheiten wie auch alles einzelne Weltliche emaniert sind, demgemäß $\mathrm{da} B$ alles zuvor in ihm enthalten war. Vgl. Pap. Ani (Ausgabe von Budge, The Book of the Dead, Papyrus Ani, rgr3) Pl. 28, Z. 15: ink sı p3int ntruy wollen: „Ich bin der Gott Schu der. Urzeit der Götter"! Aber, wie gesagt, ich will hier diese Frage nicht zur Entscheidung bringen, sondern nur; weil dazu genötigt, darlegen, daß ein Problem vorliegt. Ich will in diesem Zusammenhange auch ganz dahingestellt sein lassen, wie die Vermutung von BUDGE, der im lubrigen die beiden Wortbilder $p s \underline{d} t$ und p3avt nicht reinlich auseinander halten will, fundiert ist, daß die Grundbedeutung von p32yt die "Urmaterie" sei, aus welcher Welt und Götter geformt seien. Jedenfalls ist aber, auch wenn man in obigem Satze einfach übersetzt "dessen Leib der Urgott ist", doch mit dieser Interpretation noch nichts über die dem Gedanken innewohnende ägyptische Vorstellung gesagt.

Das führt mich sogleich auf GRAPOWs Polemik gegen meine $\mathrm{Zu}-$ sammenstellung des Gottesnamens Atum mit dem Worte $t m$. Wenn sich G. über die Ableitung des Gottesnamens Tum oder Atum von $\sqrt{t m}$ und über meine Bemerkung zu deren Doppelbedeutung mit Gegensinn 
ereifert (S. 206), so habe ich hierzu zu sagen, daß diese Deutung nicht von mir stammt, sondern daß sie, was G. wissen sollte, von dem Ägyptologen A. MORET in vollem Umfange vertreten wird; vgl. dessen Mystères Égyptiens (Igr3), p. Iro; wie ja denn auch tatsächlich schon älteste Texte das Wortspiel mit $\sqrt{t m}$ lieben, z. B. Pyr. I298 b coll. I 299 c; ähnlich Pap. I, 350 Leyden nach Gardiners Publikation in Ägypt. Zeitschr. 42, S. 33. Was aber in aller Welt berechtigt gerade Herrn GRAPOW dazu, gegen meine und MORETs Zusammenstellung des Gottesnamens Atum mit $\sqrt{t m}$ Stellung $z u$ nehmen? Ist doch eben GRAPOW, wie K. SETHE gezeigt hat (Zeitschr. f. ägypt. Sprache, 54. Bd., S. 40-49), bei seiner Herausgabe von Totenb. Kap. 7 nicht imstande gewesen, die dem Verbalstamm tm $=$ vollständig sein zugehörigen Wortbildungen von dem Gottesnamen Àtum zu unterscheiden! Gerade Grapow hat an mehreren Stellen den Gottesnamen Atum gelesen, wo tatsächlich das Wort tm "alles" vorliegt, und er hat sogar die innerhalb der ägyptischen religiösen Terminologie ganz unmögliche Wendung "der Herr Atum" entdeckt, während die Wortverbindung $n b$ tm " "der Herr des Alls" bedeutet I.

Ebenfalls mit unzulänglicher Anempfindung an das, religiöse Stratum hängt zusammen, was $\mathrm{G}$. anläßlich meiner Besprechung des ägyptischen Priestertitels it nt $\underline{r}$ sagt. Ich habe denselben "heiliger oder geistlicher Vater" übersetzt, während G. bei der Übersetzung "Gottesvater" mit Nachdruck stehẹn bleibt und nicht einzusehen erklärt, weshalb diese seine Übersetzung unmöglich sei. Hiebei kommt es nun freilich zum nicht geringen Teil auf das Sensorium für die religiöse Empfindung und Vorstellung der alten Ägypter an. Gegenüber der verunglückten Polemik G.s begnüge ich mich hier, festzustellen, daß ich mich in Übereinstimmung mit ERMAN befinde, der in richtiger Erkenntnis der Unmöglichkeit des Terminus "Gottesvater" als Bezeichnung für eine - Priesterklasse den in Rede stehenden Ausdruck it nt.r mit "heiliger Vater" und "göttlicher Vater" wiedergibt ${ }^{2}$. Diese Übersetzung ist durchaus einwandfrei und sinngemäB. Ich gehe nur einen Schritt weiter und erhebe die Frage, weshalb diese Wortverbindung gewählt ist. Ich werde dabei auf die Vermutung geführt, daß hier dieselbe Bedeutung von $n \underline{t} r$ vorliegen möge, wie wir sie sonst oft genug antreffen und nach welcher der Titel eigentlich besagt: Vater mit $n \underline{t} r$, mit einer bestimmten über-

I Vgl. im übrigen die oben erwahnte Abhandlung von K. Sethe in Zeitschr. f. ägypt. Sprache, 54. Bd., S.1-15.

2 A. ERMAN, Agypten und agyptisches Leben, S. $17 \dot{6}$ und 256 . 
sinnlichen Ausstattung, eben mit einer solchen, wie sie in höchstem Maße die "Götter" besitzen.

Und damit kommen wir zur Hauptsache!

Meine Behauptung gipfelt darin, daß $n \underline{t} r$ eine Bedeutung hat, die etwas ganz anderes besagt als unser Begriff Gott - wobei ich, nebenbei bemerkt, betone, unser Begriff; denn auch das deutsche Wort "Gott" hat seine Sprach- und Begriffsgeschichte. Auf demnächst über diesen Gegenstand zu erwartende belangreiche Forschungsergebnisse von RUDOLF MUCH möchte ich schon jetzt aufmerksam machen. Und ich behaupte weiter, daß nutr neben den Göttern des polytheistischen Pantheons auch den einen einzigen Gott bezeichnet und nicht,

I Auf die im obigen Text nicht besprochenen Einzelheiten in Grapows Artikel sei hier das Nötige erwidert. Was G.s Anmerkung (S. 207) ad Geb = Erde will, ist mir ganz unverstandlich. Es handelt sich bei mir keineswegs um „eine völlig verkannte Schreibung des Erdgottes $\mathrm{Geb}^{u}$. Denn es steht das Wortbild dieses Gottesnamens deutlich da - wie ich doch ganz unmißverständlich gesagt habe; dennoch bedeutet dasselbe im Zusammenhang jener Inschrift nicht den Erdgott, sondern die materielle Erde. Ich verweise dafür auf die in meiner Abhandlung angeführten Artikel der Ägypt. Zeitschr. Ich füge hier bei, daß auch andere Himmelskórper sowie der Himmel als nter determiniert werden. Der Himmel z. B. in junger (ptolem.) Zeit HorRack, Les lamentations d'Isis et de Nephthys, p. 6: „Dem Himmel gehört deine Seele, der Erde dein Leib“, wo Himmel, Seele und Leib durch den sitzenden Gott determiniert sind und wo der physische Himmel gemeint ist, wie aus dem Gegensatz hervorgeht. Der Mond als nter det. findet sich z. B. in einem Text aus der 22. Dyn.: „Die Erde ist in nächtlicher Finsternis, während der Mond $4-8)$ sie erleuchtet" (S. Birch, Medical Papyrus with the Name of Cheops, Agypt. Zeitschr. I87I, S. 62). - Die erste Stelle, an der in meiner Abhandlung diè p) unterzieht G. S. 20I einer Besprechung. Mir war das Mißgescbick begegnet, da $B$ ich in meinen Vorarbeiten die Hieroglyphe des $b$-Vogels undeutlieh schrieb und hernach als $p_{\text {; }}$ Vogel las, woraus sich mein Textbild erklärt; ich muß jedoch betonen, daß diese Stelle, die $z \mathrm{u}$ den einleitenden Satzen gehört, fur meine These gar keine Bedeutung hat. - $\mathrm{Da} B$ ich in Pyr. $376 \mathrm{f}$. das wn-Zeichen für ${ }_{n}$ genieBen " nahm, geschah auf Grund dessen, daß tatsächlich oft $\mathfrak{w n}$ für $\mathfrak{w n m}$ steht, freilich sonst mit beigefügtem Det. - Uber die Erkennung und Bedeutung des Weihrauchgefäßes (G., S. 20I) besteht mir überhaupt kein Zweifel. Schlangenstabe kommen in den Totentexten in großer Zahl und in mancherlei Art vor. Wenn diejenigen Tierstäbe, welche neben den metallenen neterty zur Mundöffnung benutzt werden, wirklich durchgangig(?) Widderkojfe zeigen sollten, was die Abbildungen jedenfalls nicht klar erkennen lassen, so hätte $G$. nicht zu bemerken versäumen durfen, daB sich in einigen Fällen auf dem Widderkopfe solchen Stabes noch die sitzende Uräusschlange befindet, wodurch dás Verstandnis des Wesens eines solchen Stabes an die Hand gegeben ist. - G.s Behauptung, . daß ỉm,hnzw.stets mit „der Würdige “ ubersetzt werden müsse (S. 203) und nicht "Gefolgsmann" heißen könne, ist falsch. Letztere Bedeutung, freilich in etwas anderem Sinn als $\xi m s_{i j}$, ist sehr oft die gebotene, worüber sich G. aus dem ausführlichen Aufsatz von A. MORET "La condition des Féaux dans la famille, dans la societé, dans la vie d'outre-tombe im Recueil de trav. rel. à la phil. et à l'arch. Egypt. et Assyr. XIX (I897), Pp. II 2-I 48 überzeugen kann. 
minder das übersinnliche göttliche Kraftfluidum, das in dieser und jenèr innerweltiich-sinnenfälligen Erscheinung seiner Wirkung nach hervortritt und das auf menschliche Personen wie auf Naturobjekte übergehen, bezw. angeeignet werden kann (in Sonderheit von Verstorbenen). Und ich behaupte ferner, daß diese letztangegebene Bedeutung von 'neter sehr alt ist, älter höchstwahrscheinlich als die Anwendung dieses Wortes zur Bezeichnung eines Gottes unter den vielen anthropopathischen Göttergestalten des Pantheons. Gegen diese meine Aufstellungen hat G. nicht ein einziges sachliches Argument beigebracht; denn daß er einfach die Übersetzung der Beweisstellen nach der herkömmlichen Auffassung von ntwr meiner Übersetzung entgegenstellt, ist kein Gegenbeweis, sondern lediglich der Beweis dafür, daß ihm eine Beweisführung im Sinne seiner Auffassung unmöglich ist. Ich begnüge 'mich jedoch' nicht mit dieser einfachen Feststellung, sondern ich werde in aller Kürze den danebengreifenden Einwendungen G.s Rechnung tragen.

Den Pyramidentexten ist eigentümlich, daß sie zwei Arten von „Göttern", von neter-Wesen kennen, nebeneinander und einander gegenüberstellen. Ich habe in meiner Abhandlung, um ihren Umfang nicht zu sehr auszudehnen, absichtlich nur wenige der Belegstellen für meine These angeführt und besprochen. Im Folgenden werde ich einige damals nicht berücksichtigte Textstellen heranziehen. Mit einer solchen beginne ich jetzt. Pyr. $969 \mathrm{a}, \mathrm{b}(=$ Pepj Igo, Igr): in Ḥrzy si.k mś.n.k nn rdj.f Ppj pn tp mty d.f śs mm ntwry nțruy "Dein von dir gezeugter Sohn Horus setzt diesen Pepi nicht an die Spitze der Toten, sondern er stellt ihn mitten unter die göttlichen Götter". Es gibt Götter, welche in Wahrheit diesen Namen verdienen, ntrup, die wirklich $n \underline{t} r$ sind - wie unser Text schreibt: 979 welche 90 sind. Von diesen eigentlich und wahrhaftig so $z u$ nennenden Göttern sind andere $z u$ unterscheiden, welche zwar auch Götter 797 genannt werden, aber doch nicht 70 sind. Ich erörtere hier nicht die weitere Frage, sondern lasse es, als für unseren augenblicklichen Zusammenhang gleichgültig, dahingestellt, ob die letzteren an dieser Stelle in den zuvor genannten "Toten" zu erblicken sind oder nicht. Jedenfalls liegt hier-deutlich die Unterscheidung von zweierlei $n \underline{t} r 2 y$ vor ${ }^{\mathrm{I}}$. Es ist nun klar, daß die

I Statt der Worte $n t w w$ ntriw hat der Abschreiber obigen Textes für die Pyramide des Neferkare, des letzten Königs der VI. Dyn., i', lnw.w. f ntrw geschrieben. Nahm er vielleicht an der ihm rorliegenden Wendung Anstob" Doch wir wissen, leider nicht, ob seine Zeitschr.f. d. alttest. Wiss. Jahrg. 38. $1919 / 20$. 
Wortverbindung „göttliche Götter" eine religionsgeschichtliche Erklärung verlangt. Es würde freilich einen erträglichen Sinn ergeben, wenn man das Adj. ntir durch "stark" wiedergeben wollte; indessen sicherlich würde, falls nichts anderes gesagt werden sollte, hiefür nicht $n t r$, sondern ein anderes Wort für "stark" nach nțr gewählt sein, selbst wenn ntar tatsächlich die Bedeutung "stark" haben könnte. Das Adj. $\ \circlearrowright$ besagt vielmehr hier in ähnlicher Weise wie Pyr. I207 a (Ppj 419), wo Horus als $b j k \uparrow \bigcirc$ (göttlicher Falke) bezeichnet ist (ähnlich Totb. Kap. 78, I9 nach Pap. Nu, ed. BUDGE, p. I68: Der Tote ist bjk ntrjj), eine besondere Beschaffenheit, wie sie Wesen eignet, die irgendwie über die sinnliche Seinssphäre erhaben sind. So faßt auch BUDGE die letzterwähnte Pyramidenstelle auf: "a hawk having the quality of neter". I Auf der Grundlage der hiemit angedeuteten Anschauungsweise versteht es sich, wenn von den Göttern- im allgemeinen, die vielfach von recht vergänglicher Art sind, solche Götter unterschieden werden, die wahrhaft über neter verfügen und volle neter-Beschaffenheit haben, die ntriv ntriv..

Wenn der Tote im 79. Kap. Totb. von sich sagt: „Ich bin rein " geworden, ich bin neter geworden, ich bin hhy (Bhhzv) geworden, ich bin stark geworden, ich bin ein $b 3$ (eine mächtige Seele) geworden", so ist hier ein ProzeB ins Auge gefaßt, durch den der Verstorbene eine Reihe von Qualitäten erlangt hat, die für die Art seiner Fortexistenz von entscheidender Bedeutung sind, und unter diesen die Neter-Qualität (vgl. den aus Totb. Ig bekannten „Kranz der Neter-Qualität" nach dem

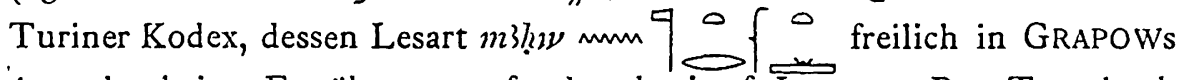
Ausgabe keine Erwähnung gefunden hat! cf. LEPSIUS, Das Totenbuch der Ägypter, Taf. XIII, Z. I5), und wieder werden wir mit "Gott", "göttlich", "Göttlichkeit" den Sinn nicht zutreffend wiedergeben können. Ganz ähnlich steht es, wenn Pyr. 759 zu dem verstorbenen und verklärten Könige gesagt ist: „Du bist beschützt, versehen mit nțr und ausgerüstet (angetan) mit der Gestalt des Osiris".' Selbst G. wird, scheint mir, nicht übersetzen wollen „Du bist versehen als Gott und

Vorlage dem Pepi-Texte glich; es ist sonach nicht unmöglich, da $B$ er die ältere Fassung bietet. Er schrieb: ${ }_{n}$ Horus hat den Neferkare nicht an die Spitze der Toten gesetzt, sondern an die Spitze seiner gottlichen Jachu". Das letzte Wort kann als "Verkläte" oder auch allgemeiner als "Lichtwesen" gefaßt werden, als in himmlischer $\delta o ́ \xi \alpha$ befindliche, $\delta o-$ $\xi \alpha \sigma \vartheta \xi \nu \tau \varepsilon \varsigma$, aber auch als ein bestimmter Kreis von Göttern, zu denen (nach MASPERO und Chassinat) die mojjw Frirw, die vier Horuskinder gehörten, wozu vgl. Emile Chassinat,

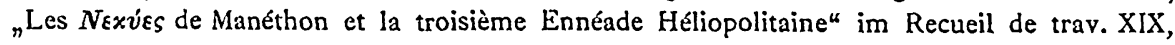
pp. 23-3I.

I E. A. Wallis Budge, The Gods of the Egyptians, I, p. 72. 
ausgerüstet als die Gestalt des Osiris". Der Text fährt fort: „auf dem Throne des Hुntj-imntjwy (d. h. des Osiris als des „Ersten der Totenreichsbewohner"), du machst sein Sein, welches er macht (d. h. du betätigst sein Wesen) unter den $i 3 h$ hy den unvergänglichen. Dein Sohn steht auf deinem Throne, ausgerüstet mit deiner Gestalt, und betätigt dein Wesen vor dem Ersten der Lebendigen nach dem Befehl des Ra, des großen Gottes". Wieder und wieder muß ich betonen, daß es G. nicht im mindesten gelungen ist, einen greifbaren Grund gegen meine Auffassung vom $n \underline{t} r$, wenn es in einer Verbindung wie hier vorkommt, beizubringen. Er beharrt einfach bei der traditionellen Bedeutung des Wortes, die in den Lexicis steht, und begnügt sich zu sagen: „Pyr. 759 wird wohl das Wort für Gott gemeint sein" ! Und was soll die Notiz, daß Pyr. $765 \mathrm{c}$ wahrscheinlich das Verbum „göttlich (rein) sein" vorliegt? Daß es sich hier um das Verbum handelt, ist nicht unmöglich, wie ich ja auch $765 \mathrm{~b}$ das Verb $n \underline{t} r$ angenommen habe (S. I72). Aber damit ist doch rein gar nichts an der Frage geändert, mit der wir es hier zu tun haben, was nämlich der Sinn von $n \underline{t} r$ ist, sei es als Verb oder Substantiv oder Adjektiv gebraucht. "Göttlich sein" dekretiert G. und läßt es mit diesem scheinbar infalliblen Spruche genug sein. Hiermit sowie mit seiner Behauptung, ein Neter-Fluidum existiere nicht, kann die Diskussion nicht gefördert werden. Was ntrr eigentlich ist, das wird, wie mir scheint, nicht übel illustriert durch jene Szene aus der Io. Nachtstunde des Im-Duat-Buches, welche die mit der Klinge nach oben aufgestellte Axt, das $n \underline{t} r$-Symbol, zeigt. Auf der Klinge ruht der Sonnenball, zu beiden Seiten sitzen die Göttinnen $N \underline{t \underline{t}}$ und Knjt, deren erstere die Axt, letztere die Sonne mit charakteristisch ausgestreckter Hand berühren. Die Situation ist klar. Die Sonne, die sich in dieser Nachtstunde anschickt, als Hprj neu zum Tageshimmel empor zu steigen, wird auf verschiedenen Wegen mit Kraft ausgestattet, vor allem mit Neter, auf dessen Substanz sie ruht. Auch der Name dieser Axt, Sdfjt $\underset{x=9}{\rightleftarrows} 4$, zeigt vielleicht diesen Vorgang an. Denn er dürfte von dem von CHABAS ${ }^{\mathrm{I}}$ erwähnten Worte $\underset{2 \approx}{\rightleftarrows}$ abzuleiten sein, demgemäß etwa "Emsigkeit" oder „Energie" bedeuten und besagen, daB diese F-Axt, auf welcher die Sonne ihre Rast vor der Wiederaufnahme ihreŕ Bahn

I Diese Bedeutung von $s d f$ ist mir nur bekannt aus F. СнавAs, Voyage d'un Égyptien en Syrie, en Phenicie, en Palestine etc. au 14. siècle avant notre ère (1866), p. 153, wo für das aus LEPsius, Denkmäler III, S. 140, c. 15 entnommene Wort der Sinn bestimmt wird. Man könnte freilich auch auf dic Wurzel śdf ${ }_{z}$ zurichten, ausrüsten ${ }^{*}$ zurückgehen. 
hält, dic für den Sonnenlauf nötige Energie in sich enthält, so dą dicselbe auf die Sonne übertragen wird. Das aber ist's, was ich mit Neter-Fluidum meine, und dies Neter-Fluidum, dieses neutrische neter, existiert in der ägyptischen Anschaung.

Auch die zweite wichtige Stelle Pyr. 752 glaubt G. mit der Bemerkung erledigen zu können, meine Übersetzung sei falsch, und durch bloße Hinzufügung: " daß übersetzt werden muß: . . du bist mächtig als ein Gott, als wärest du der Stellvertreter des Osiris". Diese Übersetzung war mir natürlich bekannt, denn sie ist gelegentlich von ERMAN gegeben worden. Ich will nicht in eine Prüfung des Wortsinnes des hier stehenden iśtj oder śtj eintreten, sondern gern annehmen, daß die Bedeutung "Stellvertreter" sich bei der hier angewandten Schreibung des Wortes rechtfertigen läßt; wennschon andere Ägyptologen wie MASPERO und BUDGE diese Bedeutung nicht anwenden, sondern das Wort mit "Sitz" oder "Platz" übersetzen. MASPEROs Übersetzung "comme le Dieu qui est à sa place, c'est-à-dire Osi.is" will wegen der willkürlichen Einfügung des Possessivpronomens sa nicht recht stimmen. Bleiben wir nun bei der Bedeutung "Stellvertreter", so wird dadurch -meine Auffassung nicht weniger möglich, sondern vielmehr m. E. die einzig mögliche: Du verfügst über neter (bemächtigst dich des n.) wie der Stellvertreter des Osiris" I.

Zur Vervollständigung meiner früheren Àusführungen sei eine treffliche Parallele zu den alttestamentlichen El-Bergen, -Hügeln, -Zedern erwähnt. Im Pap. Harris I, 7, 7 heißt es: „Ich pflanzte Weihrauchbäume in deinem Tempelhof; $\underline{d} r \quad r k \nu n t \underline{r} r$ sah man so etwas nicht wieder". "Seit der Gotteszeit": diese öfters wiederkehrende Wendung heißt doch

I Ich verweise fur den Sprachgebrauch auf Pyr. \$24a, wo nttr. im persönlichen Sinn gebraucht und vọ der Göttin Nut gesagt wird: „Die du herrschest uber die Gótter und ihre Kas", oder Pyr. $853 \mathrm{c}$ nach Merenre 385 und Neferkare 657: „Komme, der du herrschest uber (in Besitz nimmst) den Horizont, der du herrschest uber die Gotter ${ }^{\text {" }}$ ( $\$ \mathrm{hm}$

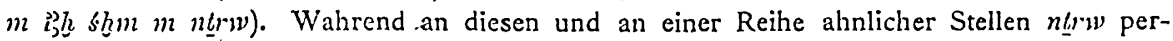
sonliche Gotter bezeichnet, kann $n t r$ in Pyr. 752 nicht in diesem Sinne gefaßt werden, man mußte denn übersetzen- ${ }_{n}$ du bist machtig uber den Gott " ; dieser nicht genannte und nicht zu nennende Gott konnte dann nur Osiris sein, und von dieses Gottes Stellvertreter auszusagen, er sei machtiger als der Gott, oder gar, er habe den Gott in seiner Gewalt, das war wohl auch innerhalb.der agyptischen Anschauung ein unvollziehbarer Gedanke. Nach alledem kann nun aber auch das erste Glied des in Rede stehenden Spruches nicht (mit ERMAN) ubersetzt werden: "Dein verklarter Geist und deine Kraft hommen zu dir", sondern es ist - wie ich úbersetzt habe - mit MAsPero zu ubersetzen: „Du bist gekommen und bist verklart", und dann ist fortzufahren: ${ }_{n}$ und nimmst das Neter in Besitz" dem Sinne von Pyr. 759. 
gewiß nicht: "seit den Tagen, da Gott auf Erden war" - obwohl, wie ich an einer Reihe von Beispielen gezeigt habe, dieser monotheistische

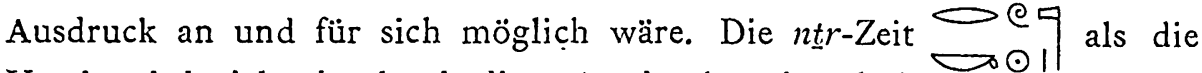
Urzeit wird vielmehr durch diese Ausdrucksweise als jene Zeit bestimmt werden sollen, in der, weil sie des nțr ais der übersinnlichen Kraftund Segensfülle mächtig war, alles einzelne, unmittelbar aus der einen übersinnlichen Urseinsquelle fließend, so herrlich wuchs, wie später nie wieder. Ich bin mir natürlich vollkommen dessen bewußt, daß ja im einzelnen Falle auch ein bestimmtes Einzelglied des Pantheons unter dem $n t r$ verstanden werden kann, auf dessen Zeit zurückgeblickt wird, in erster Linie der Gott $\mathrm{Re}^{c}$, an dessen Regierungszeit, da er König über Ägypten war, gedacht sein kann. Diese Auffassung dürfte sich um so mehr zu empfehlen scheinen, wenn man die Phrase $\underline{d} r r k z$ ntr als eine Weiterbildung der kürzeren $\underline{d} r n \underline{t} \underline{r}$ ansieht, die sich in der von SETHE besprochenen Inschrift im Grabe des Rech-mi-re zu Schech Abd el Gurna flndet. Es fügt. sich trefflich in den Zusammenhang, wenn die Ausübung der Gerechtigkeit, die "seit dem Gotte" als vom Vezier zu beobachtendes Gesetz gilt, nach SETHEs Erläuterung (Untersuchungen zur Geschichte und Altertumskunde Ägyptens, herausgegeben von K. SETHE, V, 2, Igog, S. 28) durch den Gott Thot vorbildlich geleistet sein soll und demnach $\underline{d r} n \underline{n} r$ "seit dem Gotte Re" bedeutet, unter dem Thot das Amt des Veziers bekleidet haben soll. Wo sich indessen eine solche direkte Beziehung nicht aus dem Zusammenhang ergibt, sind wir, wie mir scheint, bei dem Ausdruck rkı $n \underline{t} r$ auf die von mir vorgeschlagene Deutung als paradiesischer Zeit angewiesen; und es ist im übrigen sehr wahrscheinlich, daß der kürzere Ausdruck $\underline{d} r n \underline{t} r$ aus dem längeren $\underline{d} r$ rkzy $n \underline{t} \underline{r}$ entstanden ist, vielleicht zu einer Zeit, als der ursprünglichè Sinn dieses nicht mehr verstanden wurde.

Soll ich auch noch ein Wort zu der Beziehung des Plurals $n t r u y$ auf einen einzelnen Gott sagen? G. hat gewiß Recht mit seiner Übersetzung der Mastabastelle, und die Auseinandersetzung über den richtigen Sinn von Totb. I5 nach NAvil.le II, 2, 3 (Ba) würde hier $z u$ viel Raum beanspruchen; „des ihn kräftig Machens bei der Neunheit des Osiris-Neteru" ist ja wohl jedenfalls keine an sich unmögliche Fassung. Jedoch prinzipiell muß der betreffende Gebrauch von $n \underline{t} r v \nu$ an

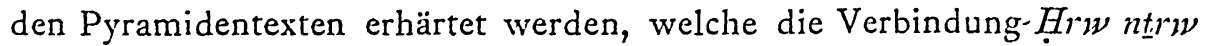
bieten. Mit der traditioncllen, auch von G. festgehaltenen Übersetzung „Horus der Götter" hat $\mathrm{m}$. W. noch niemand cinen erträglichen 
Sinn zu verbinden vermocht - ebensowenig wie mit der analogen "der 'Horus der Herrschenden" für Hrw h̆le32y - und das muß zum mindesten gegen sie stutzig machen. Nehmen wir nun Pyr. 98I ff. (Spr. 479) und Pyr. I408 ff. (Spr. 563). Es ist der wundervolle Psalm, der anhebt:

${ }_{n}$ Machet die Tore des Himmels auf und die Türen des Firmaments weit für den $\operatorname{tr} r \underline{\nu} n \underline{t} r w$, daB er hervorgehe beim ersten Tagesgrauen, nachdem er sich gereinigt in den Gefilden von Jaru!

Machet die Tore des Himmels auf und die Türen des Firmaments weit fur den F.trw des Ostens, daß er hervorgehe beim ersten Tagesgrauen, nachdem er sich gereinigt in den Gefilden von Jaru!

Moge doch hervorgehen, der hervorgeht mit dem ersten Tagesgrauen, nachdem er sich gereinigt in den Gefilden von Jaru!

Möge hervorgehn $H_{i} \cdot w$ ntrw mit dem ersten Tagesgrauen, nachdem er sich gereinigt in den Gefilden von Jaru !

Die Verdeutschung von Ḧrı ntrıy mit „Horus der Götter" stünde keineswegs, wie G. meint, in Parallele mit „Herr der Götter", nicht einmal mit „Gott der Götter", denn der Gedanke erfährt jedesmal eine . besondere Wendung. $\mathrm{Zu}$ beachten ist, daß in Pyr. 1086 a statt Merinre pn Hrin ntrin (was MASPERO übersetzt: "dieser M., der Horus die Götter") oder Neferkare ${ }^{c}$ p "N. ist Horus-Neteru" und "er ist Horus-Neteru", d. h. Horus der Gott, der von SETHE beigegebene "ältere Text" bietet ink Hriv $p$ nțriv, was nicht anders wiedergegeben werden kann als entweder „ich bin Horus, der Neteru" (Horus-Neteru) oder "ich Horus bin der Neteru", allenfalls, wenn man eine Trennung der "beiden eng zusammengehörigen Wörter hier für möglich hält: „ich bin der Horus-Neteru“. (Vgl. dazu K. SETHE, "Der Nominalsatz im Ägyptischen und Koptischen" im 33. Bande der Abhdl. d. philol.-histor. Kl. der säch's. Ges. d. Wiss. Nr. III, S. I38, I4 I und I42.) Es bleibt sonach ungezwungen keine andere Erklärung als die von mir gegebene: Der Plural steht hier im Sinne von Allgott, Gott $k . z$. Und wenn G. keinen Ägyptologen weiß, der diese Auffassung zuversichtlich bejaht, so bin ich immerhin in der Lage, ihn darauf zu verweisen, daß BUDGE die Vermutung ausgesprochen hat, daß bisweilen 999 nicht durch "Götter", sondern durch "Gott" wiedergegeben werden müssen, just as we translate the Hebrew Elohim by Lord or God $\mathrm{r}$.

I E. A. Wallis Budge, Osiris, I, p. 123, note 4. 
Darüber, daß die "Zuversicht" keine sehr verbreitete ist, braucht man sich wahrlich nicht zu verwundern, denn es fehlte eben bislang an einer Befragung der religionsgeschichtlichen Parallelerscheinungen, die erforderlich sind, um klarzulegen, welche psychologische Einstellung der religiösen Objektsvorstellung dazu führt, den Vollgehalt des singularischen Begriffs der Gottheit durch den Pluralis zum Ausdruck zu bringen.

Zum Schluß will ich noch eine Äußerung G.s besprechen, um in rechtes Licht $z u$ setzen, mit welch völliger Unschuld er allen von mir behandelten Problemen gegenübersteht. Wir gewahren hier die höchst merkwürdige Tatsache - die sich hier und da sogar als System breit zu machen strebt - daß jemand, dem die allereinfachsten Voraussetzungen für die Erörterung religionsgeschichtlicher Fragen fehlen, dennoch in anmaßendstem Tone zu solchen und über solche das Wort ergreift. Wie von der hohen Warte des Alleinwissenden herab wendet sich G. mit einem ironisch sein sollenden, in der Tat aber eine erschreckende Unwissenheit verratenden Seitenblick dagegen, daß ich die Schlange ein besonders el-haltiges Tier genannt habe. Ahnt er denn gar nicht, daß ich mich hier auf eines der wenigen ganz sicheren Ergebnisse beziehe, die bisher der allgemeinen und vergleichenden Religionswissenschaft überhaupt beschieden sind? Weiß er nicht, daß es längst außer jeder Diskussion steht, da $\dot{\beta}$ die Schlange das in vielen Religionen bedeutsamste ${ }_{0}$ Tier ist, daß sie, die zwar als Herrscher über alle anderen Tiere gedacht ist, diese Rolle empfangen hat, weil man in ihr außergewöhnliche' und durch Erfahrung und Beobachtung unkontrollierbare Kräfte wirksam sah, und daß nur noch darüber gestritten wird, ob sie ursprünglich als Seelentier galt (WUNDT) oder als stete Erneuerin ihres eigenen Leibes und von hier aus als Repräsentantin der Unsterblichkeit und Inhaberin der Verjüngungs- oḍer Unsterblichkeitskraft oder als Besitzerin eines geheimnisvollen und zugleich unheimlichen Wesens, worauf HOPFNER I den Hauptnachdruck legt, und in dieser Hinsicht als der Inbegriff dämonischer Gewandtheit, Geschwindigkeit und Plötzlichkeit? Ist Herrn Dr. G. unbekannt, daß und weshalb es kein Schlangen beherbergendes Land auf der Erde gibt, in dem nicht die Schlange im Mittelpunkt eines Anschauungskreises von mystischer Intuition oder

I Th. Hopfener, Der Tierkult der alten Agypter nach den griechisch-römischen Berichten und den wichtigsten Denkmälern (Denkschriften der Wiener Akademie der Wissenschaften 57,2$)$, S. 137 ff. 
Furcht, religiöser Scheu und Verehrung steht? $\mathrm{Dab}$ sie nicht nur im Hexenkesscl der Zauberschwestern nicht fehlen darf, sondern dab selbst Mose, der kein Götzenbild geduldet hätte, nicht umhin konnte, die Schlange am Stabe aufzurichten? Und über diesen ganzen Komplex von Tatsachen, der wieder und wieder die Schlange als Trägerin übersinnlicher Kraft vorführt, setzt sich ein Ägyptologe kühl hinweg, dem die Scḥlange in seinen Texten auf Schritt und Tritt begegnet und in dessen Studienmaterial dieses Tier in den denkbar verschiedensten Beziehungen auftritt, als "Inkorporationstier" oder Symbol der uralten Hauptgöttin Nut, als Symbol zahlreicher anderer Göttinnen, als Schriftzeichen für Göttin überhaupt, als Symbol der Königsherrschaft, als Trägerin der Sonne,' als heiliges Tier, als Unterweltswesen in allen möglichen Formen und Bedeutungen, usw., usw.! Als Inhaberin geheimnisvoller Lebenskräfte, die von ihr auf Menschen, lebende wie tote, übergehen, behauptet die Schlange in der Anschauung der alten Ägypter durch die verschiedenen Zeiten ihren Platz. Die Schlange auf dem Stabe, die uns ägyptische Bilder zeigen, möchte vielleicht an die zuvor erwähnte Handlung des Mose, die Errichtung der heilkräftigen Schlange gemahnen. Der Ka eines Menschen hat, worauf schon H. VAN DER LEEUW hingewiesen hat ${ }^{\mathrm{x}}$, gelegentlich eine Schlange an Stelle des Leibes. In eben jener Io. Nachtstunde des Im-Duat-Buches befinden sich neben dem oben erwähnten Bilde, das die Sonne auf der ntr-Axt zeigt, zwei auf ihren Schwänzen stehende Schlangen, auf deren Hälsen die Sonne ruht, augenscheinlich $\mathrm{zu}$ einem ähnlichen ${ }^{-} Z$ wecke, also um durch sie mit einer Energie ausgestattet $\mathrm{zu}$ werden. $\mathrm{Zu}$ fast allen diesen Beziehungen der Schlange in Ägypten weisen Ethnologie und Religionsgeschichte Entsprechungen allenthalben nach, wobei nicht nur an die kosmogonischen Mythen oder an den strahlenden Edelstein an der Stirn einer Schlange $z \mathrm{u}$ denken ist. Mit einer leichten Zwischenbemerkung sucht $G$. dieses ihm unbekannte und unbequeme Tatsachengebiet zu diskreditieren und hinweg zu dekretieren. Jedenfalls ist das eine Methode, die in der Wissenschaft nicht einreißen darf. Derartige Methoden des Einwendens zeigen ja auch immer nur, daß man nichts Zutreffendes einzuwenden hat.

I External Soul, Schútźgeist und der agyptische Ka, Zcitschr. f. àg. Spr., Bd. ¡4, S. 62.

[Abgeschlossen den II. August 1919.] 